\title{
ISOTHERMIC SURFACES AND THE GAUSS MAP BENNETT PALMER
}

(Communicated by David G. Ebin)

\begin{abstract}
We give a necessary and sufficient condition for the Gauss map of an immersed surface $M$ in $n$-space to arise simultaneously as the Gauss map of an anti-conformal immersion of $M$ into the same space. The condition requires that the lines of curvature of each normal section lie on the zero set of a harmonic function. The result is applied to a class of surfaces studied by S. S. Chern which admit an isometric deformation preserving the principal curvatures.
\end{abstract}

1. Introduction. The classical Gauss map of a surface in $\mathbf{R}^{3}$ assigns to a point the unit normal vector to the surface. For a surface in $\mathbf{R}^{N}$ the Gauss map assigns to a point the tangent plane which may be identified with a point in a quadric $Q^{N-2} \subset \mathbb{C} P^{N-1}$.

In recent years several papers have discussed the determination of a surface by its Gauss map. The results of K. Kenmotsu [6] show that a smooth map from a Riemann surface $R$ to the 2-sphere, satisfying an integrability condition, factors through a conformal immersion

$$
X: R \rightarrow M^{2} \subset \mathbf{R}^{3}
$$

as the Gauss map. Kenmotsu's integrability condition explicitly involves the conformal structure of $R$ and a real valued function $h$ which is to be the mean curvature of $M$.

In [3] Hoffman and Osserman give conditions on a map

$$
g: R \rightarrow Q^{N-2}
$$

involving only the complex structure of $R$, which are necessary and sufficient for the map to arise as the Gauss map of a conformal immersion

$$
X: R \rightarrow \mathbf{R}^{N} .
$$

Their results essentially imply that a conformal immersion of a Riemann surface $R$ into $\mathbf{R}^{N}$ is determined by its Gauss map, provided the mean curvature is not identically zero at some point.

Since a single map as in (1.2) determining multiple conformal immersions is in general excluded, a natural question to consider is when such a map arises simultaneously as the Gauss map of both a conformal and anti-conformal immersion. This question turns out to have a simple geometric answer which requires the following definition:

DEFInITION. A surface $M \subset \mathbf{R}^{N}$ is isothermic if, locally, there exist a pair of harmonic functions $u_{1}, u_{2}$ such that the lines of curvature of each smooth section of

Received by the editors December 1, 1987.

1980 Mathematics Subject Classification (1985 Revision). Primary 53A05; Secondary 53C42. 
the normal bundle are contained in a level set $u_{j}=$ const. This is a generalization of a classical definition which can be found in [2].

THEOREM I. Let

$$
g: R \rightarrow Q^{N-2}
$$

be the Gauss map of a conformal immersion of an orientable surface $M$ :

$$
X: R \rightarrow M \subset \mathbf{R}^{N} \text {. }
$$

Then there exists an anti-conformal immersion

$$
\tilde{X}: R \rightarrow \tilde{M} \subset \mathbf{R}^{N}
$$

such that the following diagram commutes

$$
\begin{gathered}
R \stackrel{g}{\longrightarrow} Q^{N-2} \\
\tilde{X} \searrow \quad \text { ノGauss Map } \\
\tilde{M}
\end{gathered}
$$

if and only if $M$ is isothermic. The surface $\tilde{M}$ is unique (modulo similarity transformations of $\mathbf{R}^{N}$ ) provided $M$ is not totally umbilic.

There are abundant examples of isothermic surfaces. We list a few.

(1) Constant mean curvature surfaces in $\mathbf{R}^{3}$.

(2) Surfaces of revolution in $\mathbf{R}^{3}$.

(3) Constant mean curvature surfaces $M^{2} \subset S^{3} \subset \mathbf{R}^{4}$.

In addition, we note the following properties of isothermic surfaces which when combined with the above furnish more examples:

(1) $f(M)$ is isothermic if $M$ is isothermic and $f: \mathbf{R}^{N} \rightarrow \mathbf{R}^{N}$ is conformal.

(2) If $X: R \rightarrow M \subset \mathbf{R}^{N}$ is isothermic then

$$
\underbrace{X \oplus X \oplus \cdots \oplus X}_{k}: M \rightarrow \mathbf{R}^{N k}
$$

is isothermic.

In part 4 we apply the main result to a class of surfaces studied by S. S. Chern in [1]. These are surfaces of nonzero mean curvature which admit a nontrivial isometric deformation preserving the principal curvatures. We show first that these surfaces are isothermic and second that the surface $\tilde{M}$ described above has the property that is mean curvature is the reciprocal of a harmonic function.

2. Preliminaries. Let $R$ be a simply connected Riemann surface and

$$
X: R \rightarrow M \subset \mathbf{R}^{3}
$$

a smooth, conformal immersion. We assume $M$ is orientable. After choosing a complex coordinate $z=u_{1}+i u_{2}$ on $R$, the metric induced by (2.1) has an expression

$$
d s_{M}^{2}=e^{\rho}|d z|^{2}
$$

for a smooth function $\rho=\rho(z)$ on $R$. Locally on $M$ we may choose an orthonormal frame $\left\{\xi^{r}\right\}_{r=3, \ldots, N}$ for the normal bundle $N(M)$. Differentiating $\xi^{r}$ defines

$$
d \xi^{r}=-A_{r}(\cdot)+\nabla_{(\cdot)}^{\perp} \xi^{r}
$$


The terms on the right-hand side are respectively the tangential and normal components of $d \xi^{r}$. At each point of $M,-A_{r}$ is a selfadjoint endomorphism of the tangent plane. Its eigenvalues $\beta_{j}^{r} ; j=1,2$ are the principal curvatures of $\xi^{r}$. The corresponding eigenvectors are the principal directions and their integral curves are the lines of curvature. These curves exist away from the $\xi^{r}$ umbilics which are those points of $M$ where $\beta_{1}^{r}=\beta_{2}^{r}$.

The second fundamental forms are defined by

$$
\Pi_{p}^{r}(X, Y)=d s_{M}^{2}\left(X, A_{r}(Y)\right), \quad X, Y \in T_{p} M .
$$

The trace of $\Pi^{r}$ is twice the $r$ th mean curvature

$$
h^{r}=\frac{1}{2}\left(\beta_{1}^{r}+\beta_{2}^{r}\right)
$$

On $R, \Pi^{r}$ has an expression

$$
\Pi^{r}=2 \operatorname{Re}\left(\frac{\phi^{r}}{2} d z \otimes d z+h^{r} \frac{e^{\rho}}{2} d z \otimes d \bar{z}\right)
$$

The quantities

$$
q^{r} \equiv \phi^{r} d z \otimes d z
$$

define invariant quadratic differentials on $M$. Under change of complex coordinate

$$
z_{1}=z_{1}(z), \quad \frac{d z_{1}}{d \bar{z}}=0
$$

$q^{r}$ transforms according to

$$
q^{r}=\phi^{r} d z \otimes d z=\phi^{r}\left(\frac{d z}{d z_{1}}\right)^{2} d z_{1} \otimes d z_{1} ;
$$

that is

$$
\phi_{1}^{r}=\phi^{r}\left(\frac{d z}{d z_{1}}\right)^{2} .
$$

The zeros of $q^{r}$ are precisely the $\xi^{r}$ umbilics. See [5] for details.

The quantities defined above appear as coefficients of the structural equations for the immersion

$$
\left\{\begin{array}{l}
X_{z z}=\rho_{z} X_{z}+\frac{1}{2} \sum_{r} \phi^{r} \xi^{r} \\
X_{z \bar{z}}=\frac{e^{\rho}}{2} \sum_{r} h^{r} \xi^{r} \\
\xi_{z}^{r}=-h^{r} X_{z}-\phi^{r} e^{-\rho} X_{\bar{z}}+\sum_{t} S_{r}^{t} \xi^{t},
\end{array}\right.
$$

(and their conjugates) where $S_{r}^{t}$ are defined by

$$
\nabla_{z}^{\perp} \xi^{r}=\sum_{t=3}^{N} S_{r}^{t} \xi^{t}
$$

The well-known integrability conditions of (2.12) are the Gauss equation

$$
\rho_{z \bar{z}}=\frac{1}{2} \sum_{r}\left(\phi^{r} \overline{\phi^{r}} e^{-\rho}-\left(h^{r}\right)^{2} e^{\rho}\right),
$$


the Codazzi equations

$$
\left(\phi^{r}\right)_{\bar{z}}+\sum_{t} \phi^{t}\left(\overline{S_{t}^{r}}\right)=e^{\rho}\left(h^{r}\right)_{z}+\sum_{t} e^{\rho} h^{t} S_{t}^{r}
$$

and the Ricci equations

$$
\operatorname{Im}\left\{\left(S_{r}^{t}\right)_{\bar{z}}-\frac{e^{-\rho}}{2} \phi^{r} \overline{\phi^{t}}+\sum_{l=3}^{N} S_{r}^{l} \overline{S_{l}^{t}}\right\}=0, \quad 3 \leq r, t \leq N .
$$

We define the Gauss map of

$$
X: R \rightarrow M \subset \mathbf{R}^{N}
$$

following [2]. Consider the quadratic

$$
Q^{N-2}=\left\{[\zeta] \in \mathbb{C} P^{N-1} \mid \zeta \cdot \zeta=0\right\} .
$$

Here [] denotes equivalence class. Since $X$ is conformal, one has

$$
X_{z} \cdot X_{z}=0
$$

and we define the Gauss map

$$
\begin{aligned}
& g: M \rightarrow Q^{N-2}, \\
& p \rightarrow\left[X_{z}\right],
\end{aligned}
$$

where $p$ is a point on $M$ with coordinate $z$. We will also use $g$ to denote this map pulled back to $R$ via $X$. Many details and interesting properties of $g$ may be found in [3 and 4].

3. Main result. The proposition below gives simple coordinate-dependent criteria for a surface to be isothermic.

PROPOSITION 3.1. $M$ is isothermic if and only if locally there exists an isothermal parameter $z=u+i u_{2}$ with

$$
\operatorname{Im} \phi^{r}(z)=0, \quad r=3, \ldots, N .
$$

ProOF. Assume (3.1) holds and write

$$
\Pi^{r}=\sum_{i, j=1,2} L_{i j}^{r} d u_{i} \otimes d u_{j}
$$

An easy computation shows

$$
\phi^{r}=\frac{L_{11}-L_{22}}{2}-i L_{12}
$$

so that (3.2) implies

$$
\Pi^{r}=L_{11}^{r} d u_{1} \otimes d u_{1}+L_{22}^{r} d u_{2} \otimes d u_{2}
$$

and the lines of curvature are the coordinate curves.

On the other hand suppose $u_{1}, u_{2}$ are harmonic functions such that the lines of curvature are contained in a level set $\left\{u_{j}=\right.$ const. $\}$. Since the lines of curvature intersect orthogonally, $u_{1}$ and $u_{2}$ are harmonic conjugates. Define

$$
z_{1}=u_{1} \pm i u_{2}
$$


the sign chosen so that $z_{1}=z_{1}(z)$ is holomorphic. The lines of curvature are the solutions of

$$
\operatorname{Im} \phi^{r} d z^{2}=0
$$

(see [5] for details). Since $\nabla u_{1}$ (resp. $\nabla u_{2}$ ) is tangent to the level curves $u_{2}=$ const. (resp. $u_{1}=$ const.), (3.6) implies

$$
\operatorname{Im} \phi^{r} d z \otimes d z\left(\nabla u_{j}, \nabla u_{j}\right)=0 .
$$

Using

$$
\nabla u_{j}=2 e^{-\rho}\left(u_{j \bar{z}} X_{z}+u_{j z} X_{\bar{z}}\right), \quad j=1,2
$$

this gives

$$
\operatorname{Im} \phi\left(u_{j \bar{z}}\right)^{2}=0, \quad j=1,2 .
$$

The Cauchy-Riemann equations applied to $u_{1}, u_{2}$ give

$$
u_{1 \bar{z}}=-i u_{2 \bar{z}}
$$

and we find

$$
\begin{aligned}
0 & =\operatorname{Im} \phi^{r} \cdot\left(u_{1 \bar{z}}^{2}-u_{2 \bar{z}}^{2} \pm 2 u_{1 \bar{z}}^{2}\right) \\
& =\operatorname{Im} \phi^{r} \cdot\left(u_{1 \bar{z}}^{2}-u_{2 \bar{z}}^{2} \mp 2 i u_{1 \bar{z}} u_{2 \bar{z}}\right) \\
& =\operatorname{Im} \phi^{r} \cdot\left(u_{1 \bar{z}} \mp i u_{2 \bar{z}}\right)^{2} \\
& =\operatorname{Im} \phi^{r}\left(\frac{d \bar{z}_{1}}{d \bar{z}}\right)^{2} \\
& =\operatorname{Im} \phi^{r}\left(\frac{d z}{d z_{1}}\right)^{2}\left|\frac{d z_{1}}{d z}\right|^{2}
\end{aligned}
$$

Therefore

$$
\operatorname{Im} \phi^{r}\left(\frac{d z}{d z_{1}}\right)^{2}=0
$$

and by Proposition (3.1) the coordinate $u_{1} \pm i u_{2}$ is as required.

PROOF OF THEOREM I. Let

$$
g: R \rightarrow Q^{N-2} \subset \mathbb{C} P^{N-1}
$$

represent the Gauss map of

$$
X: R \rightarrow M \subset \mathbf{R}^{N} .
$$

The existence of $\tilde{X}$ is equivalent to the existence of a smooth $\mathbb{C}$-valued function $f$ on $R$ such that

$$
e^{f} X_{z}=\tilde{X}_{\bar{z}}
$$

i.e.,

$$
\left[\tilde{X}_{\bar{z}}\right]=\left[X_{z}\right] \in Q^{N-2} .
$$

The differential equation (3.14) for $\tilde{X}$ will be integrable provided

$$
\operatorname{Im}\left(\partial_{z} e^{f} X_{z}\right)=0 .
$$


Using (2.12) this becomes

$$
\begin{aligned}
0 & =\operatorname{Im}\left\{e^{f}\left(f_{z}+\rho_{z}\right) X_{z}+e^{f} \sum_{r} \frac{1}{2} \phi^{r} \xi^{r}\right\} \\
& =\operatorname{Im}\left\{\left(f_{z}+\rho_{z}\right) \tilde{X}_{\bar{z}}+e^{f} \sum_{r} \frac{1}{2} \phi^{r} \xi^{r}\right\} .
\end{aligned}
$$

Note that the structural equations (2.12) applied to the immersion $\tilde{X}$ imply

$$
f_{z}+\rho_{z}=0
$$

so we can write

$$
f=\bar{g}-\rho
$$

with $g_{\bar{z}}=0$. ( $g$ is holomorphic.) This gives for all $r$,

$$
\operatorname{Im} e^{\bar{g}} \phi^{r}=0 .
$$

Define a new isothermal coordinate

$$
z_{1}=\int^{z} e^{g / 2} d \xi
$$

Then the transformation rule $(2.11)$ for $\phi^{r}$ gives

$$
\phi_{1}^{r}=e^{-g} \phi^{r}
$$

and so (3.19) implies

$$
\operatorname{Im} \phi_{1}^{r}=0
$$

and $M$ is isothermic.

Conversely if $\operatorname{Im} \phi^{r}=0$ for all $r$ then define

$$
\tilde{X}_{\bar{z}}=e^{-\rho} X_{z}
$$

and one easily checks using (2.12) that

$$
\operatorname{Im} \partial_{z}\left(e^{-\rho} X_{z}\right)=\operatorname{Im}\left(e^{-\rho(1 / 2)} \sum_{r} \phi^{r} \xi^{r}\right)=0 .
$$

For the uniqueness of $\tilde{M}$, note that by [4] Theorem 2.3 the Gauss map of $\tilde{M}$,

$$
\tilde{g}=\left[\tilde{X}_{\bar{z}}\right]=\left[e^{f} X_{z}\right]
$$

determines the immersion $\tilde{X}\left(\bmod\right.$ similarities) provided $\tilde{X}_{\bar{z} z} \not \equiv 0$. However,

$$
\tilde{X}_{\bar{z} z}=e^{f}\left(\left(f_{z}+\phi_{z}\right) X_{z}+\sum_{r} \frac{\phi^{r}}{2} \xi^{r}\right) .
$$

The right-hand side cannot vanish identically unless $\phi^{r} \equiv 0, r=3, \ldots, N$, which is the totally umbilic case. 
4. Application to a class of surfaces. In [1] S. S. Chern classified the umbilic free surfaces $M \subset \mathbf{R}^{3}$ which admit a nontrivial isometric deformation preserving the principal curvatures. Besides the classical examples of constant mean curvature surfaces, Chern found a second class of Weingarten surfaces with the property that the conformal metric

$$
d \hat{s}^{2}=\|\nabla h\|^{2}\left(h^{2}-K\right)^{-1} d s_{M}^{2}
$$

has constant Gaussian curvature $\hat{K}=-1$. We will show these surfaces are isothermic and show the surfaces $\tilde{M}$ obtained by Theorem I have an interesting property.

THEOREM II. Let $M$ be an umbilic free, nonminimal, surface in $\mathbf{R}^{3}$ admitting a nontrivial isometric deformation preserving the principal curvatures. Then

(i) $M$ is isothermic;

(ii) If $\tilde{M}$ is the surface obtained via Theorem I, the mean curvature $\tilde{h}$ of $\tilde{M}$ satisfies

$$
\tilde{\Delta}\left(\frac{1}{\tilde{h}}\right)=0 \quad(\tilde{\Delta} \text { is the Laplace-Beltrami operator on } \tilde{M}) .
$$

REMARK. If $h \equiv$ const. on $M$ then the above result is well known. The surface $\tilde{M}$ also has constant mean curvature so (4.1) holds trivially.

Following [1], introduce an orthonormal moving frame $\left\{e_{1}, e_{2}, e_{3}\right\}$ along $M$ with $e_{1}, e_{2}$ the principal directions and $e_{3}$ the unit normal. Let $\omega_{j}$ be the dual one forms and as usual define $\omega_{i j}$ by

$$
d \omega_{i}=\omega_{i j} \wedge \omega_{j}
$$

By choice of frame we have:

$$
\omega_{13}=a \omega_{1}, \quad \omega_{23}=c \omega_{2}
$$

where $a>c$ are the principal curvatures. Next introduce the one form

$$
\theta_{1}=\frac{2 d h}{a-c}
$$

Let $\alpha_{1}$ be the symmetry of $\theta_{1}$ with respect to the principal directions:

$$
\alpha_{1}=\theta_{1}-2\left(\frac{2}{a-c} e_{2}(h)\right) \omega_{2} \text {. }
$$

Let $*$ denote the Hodge star operator

$$
{ }^{*} \omega_{1}=\omega_{2}, \quad{ }^{*} \omega_{2}=-\omega_{1}
$$

and define

$$
\theta_{2}={ }^{*} \theta_{1}, \quad \alpha_{2}={ }^{*} \alpha_{1} .
$$

The Codazzi equations on $M$ can be used to show (see [1])

$$
d \log (a-c)=\alpha_{1}+2^{*} \omega_{12} \text {. }
$$

In addition we note the important formulas

$$
\begin{aligned}
& \text { (a) } \quad d \alpha_{1}=0, \\
& \text { (b) } d \alpha_{2} \wedge \alpha_{2},
\end{aligned}
$$

which gives $\hat{K}=-1$ in the case $h \not \equiv$ const. 
PROOF OF THEOREM III. We first show $M$ is isothermic. By (4.9) and (4.10a) we have

$$
d^{*} \omega_{12}=0
$$

Locally we can define a function $\rho$ by

$$
{ }^{*} \omega_{12}=\frac{-d \rho}{2} .
$$

Define

$$
d u_{j}=e^{-\rho / 2} \omega_{j}, \quad j=1,2 .
$$

Then (4.13) together with the structural equations (4.2) imply $d d u_{j}=0$. The coordinates $u_{1}, u_{2}$ have the necessary property of Proposition 3.1 and $M$ is isothermic.

We assume by the remark that $h \neq$ const. Using (3.23) we compute fundamental quantities on $\tilde{M}$ denoted with "tilde",

$$
\begin{array}{ll}
\text { (i) } \tilde{\omega}_{1}=e^{-\rho} \omega_{1}, & \tilde{\omega}_{2}=-e^{-\rho} \omega_{2}, \\
\text { (ii) } \tilde{a}=e^{\rho} a, & \tilde{c}=-e^{\rho} c, \\
\text { (iii) } \tilde{h}=e^{\rho}\left(\frac{a-c}{2}\right) . &
\end{array}
$$

The Hodge star operator of $\tilde{M}$ gives

$$
{ }^{\tilde{*}} \tilde{\omega}_{1}=\tilde{\omega}_{2}, \quad{ }^{*} \tilde{\omega}_{2}=-\tilde{\omega}_{1}
$$

which gives using (4.15(i))

$$
{ }^{*} \omega_{1}=-\omega_{2}, \quad{ }^{*} \omega_{2}=\omega_{1} .
$$

By (4.15(iii)), equation (4.1) is equivalent to

$$
0=d^{\tilde{*}} d\left(e^{-\rho}(a-c)^{-1}\right) .
$$

Compute

$$
\begin{aligned}
d^{\tilde{*}} d\left(e^{-\rho}(a-c)^{-1}\right) & =-d^{\tilde{*}} e^{-\rho}\left[(a-c)^{-1} d \rho-(a-c)^{-1} d \log (a-c)\right] \\
& =-d^{\tilde{*}} e^{-\rho}\left[(a-c)^{-1} d \rho-(a-c)^{-1}\left(\alpha_{1}-d \rho\right)\right] \quad(\text { by }(4.9),(4.13)) \\
& =d^{\tilde{*}} e^{-\rho}(a-c)^{-1} \alpha_{1} \\
& =-d e^{-\rho}(a-c)^{-1} \alpha_{2} \quad(\text { by }(4.5),(4.18)) \\
& =e^{-\rho}\left[(a-c)^{-1} d \rho \wedge \alpha_{2}+(a-c)^{-1} d \quad \cdot \log (a-c) \wedge \alpha_{2}+(a-c)^{-1} d \alpha_{2}\right] \\
& \\
& =e^{-\rho}\left[(a-c)^{-1} d \rho \wedge \alpha_{2}+(a-c)^{-1} d \quad(\text { by }(4.9),(4.10 \mathrm{~b}))\right. \\
& =0 .
\end{aligned}
$$




\section{REFERENCES}

1. S.-S. Chern, Deformation of surfaces preserving principal curvatures, Differential Geometry and Complex Analysis, H. E. Rauch Memorial Volume, Springer-Verlag, 1985, pp. 155-163.

2. L. P. Eisenhart, A treatise on the differential geometry of curves and surfaces, Dover, New York, 1909.

3. D. A. Hoffman and R. Osserman, The Gauss map of a surface in $\mathbf{R}^{n}$, J. Differential Geom. 18 (1983), 733-754.

4. - The Gauss map of surfaces in $\mathbf{R}^{3}$ and $\mathbf{R}^{4}$, Proc. London Math. Soc. (3) 50 (1985), 27-56.

5. H. Hopf, Lectures on differential geometry in the large, Lecture Notes in Math., vol. 1000, Springer-Verlag, Berlin and New York, 1984.

6. K. Kenmotsu, Weierstrass formula for surfaces of prescribed mean curvature, Math. Ann. 245 (1979), 89-99.

Department of Mathematics, University of California, Santa Cruz, CaliFORNIA 95064 